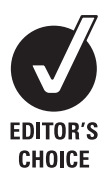

Public Health Sciences, University of Edinburgh, UK; ${ }^{2}$ University of Southampton and the Wessex Cancer Genetics Clinic, Princess Anne Hospital, Southampton, UK; ${ }^{3}$ The Ethox Centre, University of Oxford, Oxford, UK

Correspondence to: Nina Hallowell, Public Health Sciences, University of Edinburgh, Teviot Place, Edinburgh EH8 9AG, UK; nina. hallowell@ed.ac.uk

Received 8 January 2008 Revised 2 May 2008 Accepted 8 May 2008

\title{
Healthcare professionals' and researchers' understanding of cancer genetics activities: a qualitative interview study
}

\author{
N Hallowell, ${ }^{1}$ S Cooke, ${ }^{1}$ G Crawford, ${ }^{2}$ M Parker, ${ }^{3}$ A Lucassen ${ }^{2}$
}

\begin{abstract}
Aims: To describe individuals' perceptions of the activities that take place within the cancer genetics clinic, the relationships between these activities and how these relationships are sustained.

Design: Qualitative interview study.

Participants: Forty individuals involved in carrying out cancer genetics research in either a clinical $(n=28)$ or research-only $(n=12)$ capacity in the UK.

Findings: Interviewees perceive research and clinical practice in the subspecialty of cancer genetics as interdependent. The boundary between research and clinical practice is described as vague or blurred, and this ambiguity is regarded as being sustained by a range of methodological, ethical and economic factors. The implications of these findings for the "therapeutic misconception" are explored. It is argued that while research participation is seen as having therapeutic benefit for individual patients, the interviewees are not labouring under any misconceptions about the relationship between research and clinical care. It is suggested that concepts such as the "therapeutic misconception" may have less relevance in highly technological specialities that are characterised by a developing evidence base.
\end{abstract}

Research and clinical care are frequently understood as separate activities, deriving from very different motivations. Interventions carried out as clinical care are personalised; they are motivated by individual patients' needs and any risks are justified by anticipated benefits for the patient. Research interventions, on the other hand, are independent of patients' interests; they are hypothesis-driven rather than needs-driven. ${ }^{1}$ Clinical research is directed towards generating knowledge that may (but may not) benefit (future) patients, and may potentially cause some individuals harm; hence the need to ensure that participants' consent is free and informed. It can be argued that although research may not be in patients' best interests, that does not mean that all research is necessarily against patients' interests; indeed, many clinical trials compare different treatments that may be of known benefit; what is unknown is which treatment is of the greatest benefit or contains the greatest risks.

While, in theory, research and clinical care are frequently seen as highly differentiated, in practice the situation may be more complex. It has been observed that the ambiguous status of some activities that take place in the clinic, coupled with uncertainty and genuine complexity surrounding the definition of research versus clinical care, particularly in the field of genetics, creates a confusing situation for researchers/clinicians and research ethics committees (RECs). ${ }^{2}$ This arises because sometimes it is difficult to distinguish diagnostic DNA-testing undertaken in clinical contexts from molecular research carried out to characterise a set of mutations, which will incidentally provide research participants with a genetic diagnosis. ${ }^{3}$

The difficulties of distinguishing medical procedures (such as DNA-testing) carried out in research projects from those in clinical practice may have profound implications for consent. First, because of the open-endedness of some genetic investigations, the standards of consent used may be appropriate for clinical practice but fail to meet the ethical standards required by RECs for clinical research. ${ }^{3}$ Second, offering "therapeutic" or "clinical" (eg, diagnostic tests, surveillance, chemoprevention) procedures under research protocols has the potential to generate a "therapeutic misconception", ${ }^{4-8}$ whereby research participants "misunderstand" the nature of the activity they have consented to.

The concept of the therapeutic misconception was coined over 25 years ago ${ }^{4}$ to explain one of the ways in which research participants are said to "mis"perceive their research participation. The therapeutic misconception refers to those occasions when research participants "misinterpret" the goals of research and believe that research activities (such as random allocation) are primarily motivated by therapeutic intent rather than to generate generalisable knowledge. ${ }^{4-8}$ While a number of studies suggest that research participants in a variety of different types of clinical studies, ${ }^{9}{ }^{10}$ DNA-mutation searching ${ }^{11}$ and genetic epidemiological research ${ }^{12-14}$ may "misunderstand" the goals and/or methods of research" ${ }^{9-11}$ and expect a therapeutic gain ${ }^{910}$ or to obtain a genetic diagnosis, ${ }^{12-14}$ the extent to which clinicians or clinical researchers could be said to be susceptible to the therapeutic misconception is less well documented. Miller and colleagues suggest that individuals involved in providing $B R C A 1 / 2$ genetic diagnostics as part of research protocols are equivocal about the nature of the service they offer, and describe themselves as supplying a "quasi-clinical" service. ${ }^{15}$ A recent interview study of surgeons carrying out a pragmatic randomised controlled trial suggests that these clinicians failed to understand the design of the trial and were unclear about its aims and methods. ${ }^{16}$ Likewise, a questionnaire study of oncologists found that approximately $20 \%$ of medical oncologists and $38 \%$ of paediatric oncologists 
thought that the main societal purpose of clinical trials is to offer state-of-the-art treatment to individual patients rather than to create generalisable knowledge. ${ }^{17}$ These studies indicate that, on occasion, researchers, like research participants, may "misperceive", or have alternative views about, the primary aims of the activities they are engaged in.

The present study was devised to explore individuals' perceptions of the nature of the relationship between the activities designated as "research" and "clinical care" within the subspecialty of cancer genetics. One objective was to ascertain the meaning of research and clinical care from a range of lay and professional perspectives and compare their perceptions of the ways in which these activities are related. This paper reports data from interviews with a subset of the participants - those individuals who have direct experience of undertaking clinical research in this subspecialty, many of whom were also involved in providing clinical care.

\section{Why cancer genetics?}

There were two reasons for choosing cancer genetics services as the focus of this study. First, given our interest in exploring individuals' perceptions of the ways in which research and clinical care coexist within the clinic, we believed it important to focus upon an area of medicine that is characterised by a high degree of research activity. Historically, cancer genetics has been a very research-active clinical subspecialty. Many of the clinics evolved during the 1990s to provide and identify families for molecular genetic research studies, and many clinical posts were funded from research budgets. This situation still exists-for example, the CAPP2 study, a chemoprevention trial for an inherited form of colorectal cancer (HNPCC)-part funds genetic counsellors who recruit to CAPP2 and carry a clinical caseload.

Second, because we were keen to study individuals' perceptions of the ways in which the activities defined as research and clinical care interact within everyday practice, we wanted to look at a subspecialty in which these activities are less clearly differentiated. Thus, we chose cancer genetics, because many of the procedures on offer (eg, DNA testing to confirm rare cancer syndromes such as familial leiomyomatosis, or ovarian screening, which is only available in most areas of the UK through UKFOCSS, an observational study of high-risk women) are available only on a research basis. While this may change as the evidence base builds up, the status of many activities (DNA testing, bodily surveillance) in cancer genetics clinics is currently unclear.

\section{METHODS}

\section{Recruitment}

Those working in the subspecialty of cancer genetics in the UK in a clinical and/or research capacity were contacted using data available within the public domain (eg, list serves and websites). In addition, some participants were referred to the study by colleagues who had been interviewed earlier. Potential participants were sent a letter or email informing them of the study, an information leaflet outlining the study and an expression of interest form to return to the research team. Once they had noted an interest, they were contacted by the researchers and face-to-face or telephone interviews were arranged.

\section{Participants}

Of the 62 individuals invited to participate, 40 (65\%) agreed to be interviewed-13 (32\%) men and 27 (68\%) women. Their mean age was 47 years (range 32-71). All were involved in research in some capacity: 12 (30\%) of those interviewed had no direct involvement in patient care and were classified as academic researchers; the remaining 28 (70\%) were employed as healthcare professionals (HCPs). Eighteen HCPs (45\%) were clinical geneticists (consultants or specialist registrars) and 10 $(25 \%)$ practiced as genetic nurse specialists/counsellors. The research involvement of 11 (39\%) of the HCPs was limited to recruitment, and the remainder $(17 ; 61 \%)$ were involved in recruitment and generating their own research projects. Most HCPs were involved in recruiting to or undertaking a variety of different types of research projects, from psychosocial interview studies to DNA mutation searching or gene association studies, whereas the academic researchers tended to specialise in particular research areas (see table 1).

\section{Data collection and analysis}

The interviews were undertaken by SC or NH between January 2006 and March 2007. Eighteen interviews (45\%) were carried out by telephone, the majority during working hours; the remaining interviews took place face to face at a location of the participant's choice (participant's/interviewer's workplace). Interviews lasted $1-1.5 \mathrm{~h}$ and were tape-recorded with the interviewee's consent.

Interviewees were asked to provide a narrative account focusing upon their role in the organisation they worked in and their research and/or clinical practice in cancer genetics. A series of exploratory questions was used, which investigated: research experience, ethical and practical difficulties encountered in undertaking their (research/clinical) work, perceptions of the relationship between research and clinical practice and duties as a researcher/clinician and role conflicts. The interview schedule was dynamic and questions used in later interviews were developed in the light of emergent findings.

Verbatim transcriptions were obtained for 39 interviews (one tape was inaudible). These were read through many times to identify recurrent themes within and between participants' accounts. The method of constant comparison ${ }^{18}$ was used to develop a coding frame for the analysis. The emergent codes were discussed and verified by at least two members of the research team. OSR N6, a qualitative data analysis software package, was used to manage the data.

\section{RESULTS}

In general, there were few differences between the researchers' and the HCPs' responses in these interviews; on occasion, however, it was apparent that individuals' perceptions of cancer genetics activities were influenced by their degree of engagement with the different activities, and this is noted in the analysis below. As far as most interviewees are concerned, there is no hard and fast division between clinical practice and research. Indeed, many commented that these activities were indistinguishable at times. A range of methodological, normative or economic factors were seen as responsible for maintaining the ambiguous relationship between clinical care and research in cancer genetics.

\section{Dismantling the methodological divide: Research and clinical care?}

Research and clinical activities were described by both HCPs and researchers as difficult to distinguish, because they are characterised by a number of methodological similarities. Many of the interviewees constructed medical practice as an 
Table 1 Research activity or specialty of interviewees $(N=40)$ working in cancer genetics research

\begin{tabular}{lc}
\hline Activities of those interviewed & Number $(\%)$ \\
\hline Research activities of healthcare professionals $(\mathrm{n}=28)$ & \\
Clinical/epidemiology & $19(68)$ \\
Molecular science & $5(18)$ \\
Social science & $12(43)$ \\
Research coordinator & $2(7)$ \\
Statistics & $1(4)$ \\
Specialties of academic researchers $(\mathrm{n}=12)$ & \\
Clinical/epidemiology & $1(8)$ \\
Molecular science & $3(25)$ \\
Social science & $5(42)$ \\
Research coordinator & $2(17)$ \\
Statistics & $1(8)$ \\
\hline
\end{tabular}

exploratory activity, which utilises the same types of procedures or methods as clinical research:

Anybody and everybody who has clinical contact is offered an opportunity to participate in a mechanism that will further our understanding of whatever the clinical conundrum is. Because we are ignorant for the most part of most of the ways in which we either manage, or think we treat, conditions ... If one puts up a complete distinction, it implies that there is necessarily a process of clinical management that is free of research. I don't believe that exists. (H319, clinical geneticist and molecular researcher)

Others reflected on the similar motivations underpinning these activities. H325, a researcher who worked in a very research-active tertiary referral centre, observed that it is often difficult to determine the underlying motives or purposes for undertaking certain procedures - clinical need or scientific curiosity - and thus is impossible to identify a boundary between exploratory medical practice and experimental research:

\footnotetext{
... there is a grey area there between clinical practice and research, which is again, you know, what this hospital is all about. I mean this hospital is a research hospital, and, you know, it is sometimes very difficult to know, is this being done to a patient as part of a research trial, or as part of their standard clinical treatment? ... When are you running a [unclear term] gel for that patient, and when are you running it for yourself as a researcher? (H325, molecular researcher)
}

Some interviewees, like H319 AND H325, commented that clinical care and research had similar epistemological foundations-both activities were seeking to contribute to medical knowledge. Others regarded the similarities between these activities as being more procedural than epistemological. These interviewees saw experimental research as a separate, but necessary, aspect of some specialities, particularly those using new technologies:

And then you can't really do human genetics without getting involved in research [unclear words] because there is so much that's not known. (H217, clinical geneticist and molecular researcher)

\section{Working with patients' best interests in mind: research and clinical care}

For many interviewees, establishing the relationship between research and clinical care was seen as an ethical or normative issue. Thus, some HCPs commented that "good" clinical practice should involve some engagement with research:
No, I think it [research activity] makes me a better doctor. I think it affects the whole way the department runs, and I think it's better for it. (H225, clinical geneticist and molecular and clinical researcher)

These HCPs saw involvement in research as enabling them to improve their clinical skills:

\begin{abstract}
... I think that in fact there shouldn't be a boundary. I think anyone who is in clinical practice who is not doing research; they aren't in very good clinical practice. Because everything they do is open to question. There's almost nothing we do in medicine that couldn't be improved upon in some way ... I think that in fact research is not seen as integral to service, and it should be ... (H215: clinical geneticist and molecular and clinical researcher)
\end{abstract}

They argued that there should not be a boundary between these activities, because HCPs have a duty to their patients to become engaged in research and translate new research findings into their clinical practice:

I think clinical genetics is very much an $\mathrm{R} \& \mathrm{D}$ specialty. It's evolving hugely. And I think that you can't just sit back and go, no, actually I'm just an NHS geneticist. I'm not an academic, but I have all this research on the go because I think that's part of practising as a clinical geneticist. We don't do nights, we don't do weekends, we don't have a huge clinical burden. So I think this $\mathrm{R} \& \mathrm{D}$ aspect is a routine part of what we do, and it's our responsibility to our genetic patients, that we are endlessly translating research, not just in cancer but in every aspect of genetics. It's moving on, we've got to be current. And you can't really be current if you're having nothing to do with research. You can't have them as separate really (H225: clinical geneticist and molecular and clinical researcher)

In addition to the societal benefits (eg, improving knowledge about disease and making the UK more economically competitive) some potentially "therapeutic" benefits (eg, accessing a DNA test) of research participation were identified.

I think in clinical genetics one of our main roles as doctors certainly is to identify patients who could be of benefit to trials or who might themselves benefit from trials. So I mean that's part of the interface that we play ... (H238, clinical geneticistrecruiter)

Thus, it became apparent that the perceived ambiguity between research and clinical care both exists at an abstract level and is also frequently encountered, and may even be intentionally maintained, in clinical practice. Many of the HCPs cited their clinical obligations as a justification for their failure to draw a distinction between research and clinical care. This is illustrated by $\mathrm{H} 212$, who argued that participation in research may be in her patients' best interests:

I'm not sure that one needs a really crystal clear boundary. Particularly in genetics, we are constantly moving from the position of just having discovered a gene to saying, can we do it as a clinical test, etcetera? ... You have an obligation as a clinician to work out whether it's worth doing, and what information it's going to give you that you can give to the patient. (H212, clinical geneticist and clinical molecular researcher)

She described how she included an assessment of individual patients' suitability for participation in research studies as part of the weekly clinical review:

[At] our clinical meetings I view part of my role as to working out for each family that we deal with, whether there are any 
research studies that are currently going on that might be appropriate for them to be invited into, on the grounds that we have not got enough resources for cancer genetics ... If we can do nothing else for them, the minimum we can usually do is offer them a research study to participate in ... So I might invite them to a couple ... which might give them the most benefit ... (H212, clinical geneticist and molecular and clinical researcher)

As many of our interviewees observed, the blurring of the boundary between research and clinical care occurs in more than one way. By describing herself as "going to research to help ... " particular patients, H213, like $\mathrm{H} 212$, constructed research studies as catering for patients' needs; however, she observed that ambiguity also arises because research participation also generates clinical needs:

I think there are two major sites where it [the boundary between research and clinical practice] is blurred. One is where in fact the research is going to help people clinically but it hasn't yet been brought into service ... So we had to go to research to help that patient. And then the other aspect is where research throws up answers that have to be-I mean we had to deal with [a research result] clinically, because one of these women could have got cancer. (H213, clinical geneticist, oncologist and molecular and clinical researcher)

$\mathrm{H} 236$, a researcher, similarly commented that research findings, in this case the results of MRI scans, frequently need to be followed up in the clinic, and that both researchers and clinicians have a duty to ensure that this is done:

Yes I mean the results of the screening; the MRI and mammography will then become a clinical issue for the patient certainly. If anything was found it would be followed up clinically and we as the researchers will just monitor what is going on, we don't have any influence on that course if you like. So yes I guess there is definitely a blurring. You can't see something on an MRI scan and ignore it. It's got to be acted upon. (H236, researcher-epidemiology)

The above data suggest that research protocols are being utilised to provide care, and thus the unclear nature of the relationship between research and clinical care in cancer genetics is not accidental. As the next section confirms, constructing this relationship as indistinct or vague can be seen as a deliberate rhetorical strategy, for it enabled the HCPs in this study to justify their use of research studies to supplement NHS services.

\section{Accessing scarce resources: research as clinical care}

In the interviews with the healthcare professionals, in particular, research was portrayed as a valuable asset, primarily because the procedures on offer in research protocols were currently rationed or unavailable as a clinical service. Thus, like H212 above, a number of HCPs explained how participation in research studies is used to circumvent resource shortfalls in the clinic. H225, for example, described how research participation was the only way in which families could access DNA testing in the case of some rare diseases for which service tests are currently unavailable:

A lot of our patients' tests and things, actually the only way we can get them tested is through research. So that patients are taking part in research because they're one in 500000 or something, you know, and the only way you can do that is to take part in somebody's research in Germany, to get your gene looked at. (H225, clinical geneticist and molecular and clinical researcher)
As most of the genetic tests for hereditary cancers are now offered as a clinical service, interviewees more commonly talked about how participation in DNA testing studies, such as The Familial Breast Cancer Study (BRCA3), is used to overcome resource allocation problems. Some described how entering their patients into the BRCA3 study speeds up the process of mutation searching in families who are eligible, but not a high priority for, NHS service testing:

If they are eligible for testing then we give them to our [NHS service] lab as well to the BRCA3 study. And we don't say don't test them because they are in BRCA3 or anything but we get BRCA3 results before we get our own results. And it's therefore useful for speeding up the procedure because obviously if they find things then we replicate that result in our own lab very quickly given that they know what to look for and can then start testing the family. So it's very useful for the families because it achieves the same end of diagnostic testing rather more quickly than left to our own devices. (H228, clinical geneticist-recruiter)

Others described how participation in BRCA3 enabled those who do not fit local NHS criteria to access BRCA mutation screening:

Well I think some people are recruited into studies as a way of getting round this resource problem, because some studies offer something as part of the study which is not offered as part of a service. So for example, if you recruit someone into the BRCA3 study we know that they will get mutation testing for the BRCA genes as part of the study on a research basis so it is a way of getting mutation testing done for someone who you are not able to offer mutation testing to ... I think that happens quite a lot ... I mean there is another study we are involved in, an ovarian screening study for UKFOCSS, and that is another example as using the study as a way of providing a clinical service. (H2O2, clinical geneticist-recruiter)

Like H202, the HCPs in this study talked about how research is used to access surveillance technologies. For example, H208 described how women had participated in a chemoprevention trial (IBIS 1) to obtain breast screening:

\begin{abstract}
... women came into IBIS 1 to get extra mammograms, because they wouldn't get them otherwise ... And in terms of only being able to get something as part of a research study that would something like UKFOCSS, the ovarian screening study, where there is nothing on offer for them screening-wise unless they go into the research study ... the MARIBS study, again they wouldn't be able to get an MRI unless they were part of the study. And I think there's always a juggle. I mean I've had lots of issues in [place name], with breast screening, especially for the moderate-risk women. At the moment they won't screen them. And their comeback to us is, we'll only do it if it is part of a research study. (H208, genetic nurse counsellor-recruiter)
\end{abstract}

Some surveillance technologies, like $x$ ray mammography, are already offered as a clinical service, but only for certain at-risk groups, whereas others (eg, MRI mammography and ovarian screening) are not available as a routine clinical service for women at high to moderate risk in the UK because their efficacy is unproven. Our interviewees talked about how they entered women into research studies (MARIBS, Breast MRI, and UKFOCSS Ovarian ultrasound + blood serum estimation) so they could access these forms of screening for their patients:

. the MRI scanning is a ... is probably the route to go and I think also having a study of that sort is really the only way that 
you're going to get quite expensive investigations of that type resourced through the health service, so I think that that's been a valuable study to participate in. (H216, clinical geneticist and molecular researcher)

Many acknowledged that if a centre takes part in research, they can contribute valuable efficacy data and, as H216 suggests, gain access to costly resources for their patients. Indeed, many of our interviewees conceded that in the case of some studies there are good economic incentives, as well as scientific reasons, for a centre's research participation.

\section{Research or clinical care: a dynamic relationship}

The data suggest that research and clinical care are perceived as standing in a complex and interdependent relationship. Indeed, as the interviews progressed it became clear that many interviewees perceived the boundary between these activities as very flexible. Sometimes this boundary was seen, and in the opinion of some, needed to be seen, as "blurred" (H213), "fluid" (H201) or "not very clear cut" (H202, H238); at other times it was constructed as distinct, and research and clinical care were seen as very clearly differentiated:

\begin{abstract}
Well clinical practice I guess is the standard service that's offered through the NHS for patients and research is obviously you know, research is being conducted by a team who are trying to get to the bottom of some question or other and generally you know, if not aimed at benefiting the research participants necessarily although sometimes that can be the side effect. (H201, genetic nurse counsellor and social science researcher)
\end{abstract}

In their efforts to establish a difference between research and clinical care, many interviewees, like H201, once again reflected upon the epistemological foundations underpinning clinic activities. They commented that although the relationship is unclear at times, clinical care and research can be seen as different types of activity, because clinical care involves the application of pre-existing knowledge whereas the aim of research is the generation of new knowledge:

\section{Well um ... in my speciality not very clear-cut ... clinical practice should follow best practice guidelines of whatever sort. Um ... we are kind of in a moment of kind of evidence-based medicine .. when it comes to research we are moving away from that and trying to generate evidence, new evidence and testing hypoth- eses. (H238, clinical geneticist-recruiter)}

Thus, it was accepted that the aim of clinical care is to cater to individual clinical needs, while the objective of research is to discern general trends. However, although our interviewees commented upon these epistemological differences, it was also clear that they regarded the relationship between research and clinical care as dynamic and evolving. H215 described how the nature of this relationship had changed over time, observing that there had been a natural divergence as the evidence base in cancer genetics had grown over the previous decade:

\footnotetext{
... to some extent the wave of research has passed by, and most of our routine genetic patients are not part of research projects. We go looking for specific populations. So in fact the two things have kind of diverged naturally. (H215, clinical geneticist and molecular and clinical researcher)
}

Moreover, there was evidence that individuals' perceptions of the nature of the relationship between research and clinical care were, in part, determined by their degree of involvement with the different activities. Thus, a few HCPs who identified their

contribution to research as being only in recruitment regarded research and clinical care as clearly differentiated but speculated that others who had greater research involvement might have more difficulty distinguishing them:

\begin{abstract}
in my mind it's quite clear, but I suspect if I was a researcher, I might feel that it's not as clear, but that would be because I'd be doing a specific piece of research, and so the two would become integrated together, ... whilst I'll use research to influence my clinical practice, it's not part and parcel of the driver for my clinical practice, whereas if you were doing research and that requires clinical practice then the research can be the driver, or one of the drivers. (H218, genetic nurse counsellor-recruiter)
\end{abstract}

However, those who thought research and clinical care in cancer genetics were easily distinguished were in the minority. Most interviewees thought these activities were frequently indistinguishable but that at certain times attempts should be made to differentiate them, particularly for their patients, whom they reported as frequently confusing research with clinical care. Thus, the HCPs in this sample described themselves as having a duty to their patients to clarify the nature of the activities that take place in the clinic:

And I think it's very important that they [patients] know what the difference is, and when something is research and when something is offered as a clinical service, and when the information coming back from that whatever, whether it can be relied on or not. (H208, genetic nurse counsellor-recruiter)

\begin{abstract}
... the patients need to know what's clinical testing and what's research ... because with the research, they're aware that it's either going to help their family or their relatives or people in general in the future. And with clinical testing, it's just pertaining to what we can do with them at the moment and it might be limited. (H214, genetic nurse counsellor-recruiter)
\end{abstract}

As we have reported elsewhere, ${ }^{19}$ this group of interviewees provided a number of justifications for distinguishing research and clinical care for their patients and also outlined a range of practical strategies that they routinely used to maintain the boundary between these activities.

\section{DISCUSSION}

This study suggests that individuals who carry out research in cancer genetics perceive the relationship between research and clinical care as flexible, permeable and permanently shifting. The ambiguous nature of this relationship is seen as being sustained by the methodological similarities that exist between these activities and, perhaps more importantly, HCPs' need to cater for patients' needs in a clinical context that is rapidly evolving and characterised by a lack of economic resources and an inadequate evidence base.

Previous research has suggested that patients frequently confound research and clinical care, ${ }^{9-11} 19$ believing their participation to be therapeutically motivated, ${ }^{45}$ and that some HCPs involved in carrying out clinical research are similarly uncertain about the primary aims of research activities. ${ }^{15-17}$ The data collected in this study suggest that our interviewees have a complex and dynamic understanding of the activities that take place in the cancer genetics clinic. Like the earlier research, ${ }^{15-17}$ our analysis suggests that many of our interviewees see entry into research studies as having therapeutic benefits for patients. Indeed, there was evidence that they regard research participation as serving a clinical need and also as generating one that they or other HCPs have a duty to 
address by either treatment or referral for treatment. Thus, these data could be interpreted as providing support for the existence of the therapeutic misconception in this group of clinicians and researchers.

However, as Appelbaum and colleagues note, ${ }^{5}$ to maintain a therapeutic misconception one has " ... to deny the possibility that there may be major disadvantages to participating in clinical research that stem from the nature of the research process itself" (p20). As we have noted elsewhere,$^{20}$ while many of our interviewees were clearly aware of the disadvantages of research-based genetic testing (namely, non-quality-controlled DNA test results) or research-based screening (ie, the rate of false positives and thus the potential for unnecessary exploratory procedures), this did not affect their view that patients might derive personal benefit from research participation. ${ }^{15} 17$ Thus, it was not that these interviewees denied or ignored the risks inherent in research studies, but rather that they regarded the anticipated benefits (such as receiving a longed-for DNA test result or obtaining desired surveillance) as outweighing the risks. ${ }^{1}$ Such observations suggest that these interviewees are not in the grips of the therapeutic misconception per se-that is, that decisions to enter patients into research are taken under the mistaken assumption that research may have a (quasi) therapeutic benefit ${ }^{15}$ - primarily because it is clear that they do not perceive research and clinical activities as differentiated in some instances. In other words, as far as our interviewees were concerned, on occasion, and in some contexts, research is care.

To support this claim, it can be argued that the "therapeutic misconception" exists only in a world in which therapy and research are seen as distinct forms of activity. While research and clinical practice, or therapy and experiment, are frequently described as binary oppositions, as situated at either ends of a continuum of medical work, ${ }^{21}$ this study suggests that in reality there is a great deal of uncertainty regarding the point at which these activities connect (merge) or disconnect (split). These interviews suggest that those involved in carrying out research in cancer genetics perceive the points on this continuum where the activities designated as research or clinical practice fall as ambiguous and flexible. Arguably, this ambiguity may be sustained by the evolving nature of cancer genetics-more particularly, by the fact that the evidence base within this subspecialty, like that of clinical oncology, is still in the process of construction. Thus, unlike other areas of medicine, which are less research active (eg, surgery ${ }^{16}$ ) and have a more welldeveloped evidence base or a corpus of accepted practice, cancer genetics, as a relatively recently formed and highly technological subspecialty, has no traditional modes of care to draw upon. Some diagnostic procedures (DNA tests) are still in development, and other treatment and surveillance regimens are unproven. Therefore, as noted above, in some areas of the UK many "clinical" procedures are available only in the context of research protocols. Arguably, cancer genetics is a translational subspecialty par excellence. However, unlike other areas of translational medicine, sugh as embryonic stem cell research, it is characterised by a bidirectional movement-research findings are translated from the bench to the clinic, while at the same time clinical observations and experiences are used to generate research questions. In this subspecialty, individual care is frequently used to discern general trends, and the pursuit of general trends may be used to provide care. Thus, clinic activities in cancer genetics appear ambiguous, because in some cases they are actually in the process of translation from bench to bedside and vice versa.
These interviews suggest that the boundary between research and clinical practice shifts, depending upon who is describing it and why it is being described. For those interviewees whose involvement in research was confined to a recruiting role, the boundary between research and care was more clearly defined, whereas for others who both generated research and cared for patients, the boundary between these activities was more indistinct. It can be speculated that this latter group of HCPs has a personal and professional interest in portraying the boundary between research and clinical care as more flexible, not least because constructing an account in which activities designated as "research" are portrayed as having therapeutic as well as scientific benefits enables them to portray themselves as fulfilling their clinical obligations. ${ }^{22}$ However, although recruiting patients to their own and others' research projects may be constructed as altruistic or beneficent behaviour, this practice also has personal gains, for it enhances their research status and thus may boost their career prospects. Nevertheless, it must be noted that all interviewees-HCPs and researchers alike-acknowledged that how they perceived, or constructed, the relationship between research and clinical care was much less important than how these activities were presented to, and understood by, patients.

\section{Study limitations}

This study focused upon a small sample of individuals who work in a highly technological and evolving subspecialty; it is therefore possible that the view of the relationship between clinical research and practice outlined above reflects a sampling bias and is specific to those who work in the field of cancer genetics. Clearly, further research is needed to determine whether this relationship is perceived as similarly ambiguous by those who work in less technological or more established specialties.

Second, to what extent are these observations influenced by the fact that the majority of the interviewees occupy the "middle ground": ${ }^{23}$ they are neither basic scientists nor pure clinicians. As clinician-researchers, or those who are responsible for undertaking or facilitating clinical research, they inhabit a space that is arguably characterised by conflicting interests and role expectations; to what extent does this influence their views? Indeed, it can be speculated that many of the clinicians we interviewed also hold academic posts that require them to undertake research, and thus their different role demands may predispose them to minimise the differences between research and clinical care. An investigation of the views of those working in a less translational subspecialty is clearly warranted.

Finally, to what extent does the way the project is framed or the questions are presented create the ambiguity described above? In defence of our methods, it must be noted that we deliberately picked a subspecialty in which the nature of clinic activities is more ambiguous, because we specifically wanted to study how relationships between the activities of the clinic are perceived and sustained. To this end, we were transparent about our aims. Our interviewees were informed that we wanted to gauge their perceptions of the clinic's activities and how they are related, so in that respect they were primed to discuss the nature of this relationship. However, this does not differ from other research projects in which the need to obtain informed consent requires that participants are informed about the study's aims in advance. 


\section{CONCLUSIONS}

This study suggests that individuals who are involved in carrying out cancer genetics research in the UK experience difficulties at times in distinguishing research from clinical care. While the interplay between these activities may generate ethical challenges, using research protocols to provide care does provide access to resources that would otherwise be unavailable. Arguably, the perceived interdependence between research and clinical care is maintained by structural features of healthcare in the UK, such as government funding policies. However, while the boundary between research and clinical care may be influenced by fiscal policies, ultimately it is dependent upon the maintenance of a semantic distinction, which may be less applicable when it comes to describing the activities of hightechnology medicine in the 21 st century. In conclusion, it can be speculated that in highly translational subspecialties, such as cancer genetics, the differences between the activities that take place in the clinic may literally be lost in translation.

Acknowledgements: Special thanks go to all of those who took part in the ROCC study and shared their experiences with us. Thank you to Julia Lawton for her helpful comments on earlier versions of this manuscript. We also thank the staff at Southampton, without whom this research would not have been possible, and Lesley Gardner, the project administrator.

Funding: Cancer Research UK Grant (C8671/A5831) awarded to N Hallowell, M Parker and A Lucassen.

Competing interests: None.

Ethics approval: Ethical approval for this study was received from the Scotland A Multi-Centre Research Ethics Committee on 16 December 2005, Ref 05/MRE00/112.

\section{REFERENCES}

1. Chen DT, Miller FG, Rosenstein DL. Clinical research and the physican patient relationship. Ann Intern Med 2003;138:669-72.

2. Newson A, Ashcroft R. Time to untangle ethical review of genetic research. BioNews 20 September 2004. http://www.bionews.org.uk/commentary. lasso?storyid $=2280$ (accessed 19 October 2008).

3. Parker M, Ashcroft R, Wilkie AOM, et al. Ethical review of research into rare genetic disorders. BMJ 2004;329:288-9

4. Appelbaum PS, Roth LH. The therapeutic misconception: informed consent in psychiatric research. Int J Law Psychiatry 1982;5:319-29.

5. Appelbaum PS, Roth LH, Lidz CW, et al. False hopes and best data: consent to research and the therapeutic misconception. Hastings Cent Rep 1987;17:20-4.
6. Lidz CW, Appelbaum PS, Gisso T, et al. Therapeutic misconception and the appreciation of risk in clinical trials. Soc Sci Med 2004;58:1689-97.

7. Horng S, Grady C. Misunderstanding in clinical research: distinguishing therapeutic misconception, therapeutic misestimation and therapeutic optimism. IRB 2003;25:11-6.

8. Henderson GE, Churchill LR, Davis AM, et al. Clinical trials and medical care: defining the therapeutic misconception. PLoS Med 2007;4:e324.

9. Snowdon C, Garcia J, Elbourne D. Making sense of randomization; responses of parents of critically ill babies to random allocation of treatment in a clinical trial. Soc Sci Med 1997;45:1337-55.

10. Featherstone $\mathbf{K}$, Donovan JL. "Why don't they tell me straight, why allocate it?" The struggle to make sense of participating in a randomised controlled trial. Soc Sci Med 2002;55:709-19.

11. Ponder M, Statham H, Hallowell N, et al. Genetic research on rare familial disorders: consent and the blurred boundaries between clinical service and research. J Med Ethics 2008;34:690-4.

12. Busby H. Blood donation for genetic research: what can we learn from donors narratives? In: Tutton R, Corrigan 0, eds. Genetic databases: socio-ethical issues in the collection and use of DNA. London: Routledge, 2004:39-56.

13. Dixon-Woods M, Ashcroft RE, Jackson CJ, et al. Beyond "misunderstanding": written information and decisions about taking part in a genetic epidemiology study. Soci Sci Med 2007;65:2212-22.

14. Wendel D. The debate over research on stored biological samples: what do sources think? Arch Intern Med 2002;162:1457-62.

15. Miller FA, Giacomini M, Ahern C, et al. When research seems like clinical care: a qualitative study of the communication of individual cancer genetics research results. BMC Med Ethics 2008;9:4. http://www.biomedcentral.com/1472-6939/9/4 (accessed 19 October 2008).

16. Ziebland S, Featherstone K, Snowdon C, et al. Does it matter if clinicians recruiting for a trial don't understand what the trial is really about? Qualitative study of surgeons' experiences of participation in a pragmatic multi-centre RCT. BMC Trials 2007;8:4.

17. Joffe S, Weeks JC. Views of American oncologists about the purposes of clinical trials. J Nat Cancer Inst 2002;94:1847-53.

18. Strauss A, Corbin J. Basics of qualitative research. London: Sage, 1990.

19. Hallowell N, Cooke S, Crawford G, et al. Translating research into practice: health care professionals' and researchers' understanding of cancer genetics activities in the UK. Eur J Hum Genet 2008;16(Suppl 2):46.

20. Corrigan 0. Empty ethics: the problem with informed consent. Soc Health III 2003:25:768-92.

21. Bosk C. When life fails to imitate art: evidence-based therapy and the bar to innovation. Paper presented at ESRC Science in Society Workshop-Governing medical research and medical practice: what's the difference? Goodenough College London, 18 January 2007.

22. Easter MM, Henderson GE, Davis AM, et al. The many meanings of care in clinical research. Sociol Health IIIn 2006;28:695-712.

23. Wainwright $\mathbf{S}$, Williams $\mathrm{C}$, Michael M, et al. From bench to bedside? Biomedical scientists' expectations of stem cell science as a future therapy for diabetes. Soc Sci Med 2006;63:2052-64. 